\title{
REACHING AN ALIGNMENT OF PROGRAMMES OF SOCIAL AND ECONOMIC DEVELOPMENT OF A REGION AND ITS FUEL AND ENERGY BALANCE PLAN
}

\author{
V.V. DOBRODEY, L.D. GITELMAN \& M.V. KOZHEVNIKOV \\ Department of Energy and Industrial Management Systems, Ural Federal University, Russia.
}

\begin{abstract}
Developing methods of aligning such important documents as regional forecasts of social and economic development, and the regional fuel/energy balance plan remains a current challenge determined by the fact that the documents are drafted in isolation, as well as by different levels of detail in projections and a mismatch between target indicators. It is necessary to ensure the priority development of complex regional energy system relative to other industries.

Research indicates the absence of a clear logic of coordinating strategies at the regional level of government in the context of the lack of any formal or even expert assessment of balance between them in terms of resources and applicability of appropriate target indicators.

The paper aims to discuss the possibility of creating a methodology for comprehensive analysis of the dynamics of energy consumption in a region within the framework of modified fuel and energy balance models that would be acceptable for use when information is scarce or limited, when the energy balance plan must be in line with the aggregate indicators of the approved forecast of social and economic development, and both documents have to be monitored for compliance with law. The paper sets out the authors' methodological approach to compiling variants of the fuel and energy balance plan, possible errors and incomplete data in statistics as well as standards for forecasting accuracy.

Keywords: accuracy, dynamics, energy conservation, energy efficiency, forecast, fuel/energy balance plan, information, randomness, regional economy, trends.
\end{abstract}

\section{INTRODUCTION}

The International Energy Agency points to the danger posed by the lack of key data in the system of energy information [1]. The reliability of forecasts is particularly critical to such energy deficient regions as Sverdlovsk Oblast of Russia where the suggested research method was trialled. The region consumes a lot of energy, but is short of its own sources of fuel and power.

In several countries, along with Russia, law prohibits the release of statistics for energy consumption by organizations into the public domain as primary data [2]. This makes it difficult or impossible to access quantitative parameters of corporate programmes, fuel and energy consumption data for individual organizations and, as a result, for specific economic activities. Moreover, this raises a doubt about the credibility of the published data and makes it difficult to prevent the deliberate corruption of the data, including for political reasons.

Without a fuel/energy balance forecast it is impossible to substantiate energy, environmental and social and economic development forecasts [3]. The fuel and energy balance plan provides details about the consumption of fuel and energy resources, enables the analysis of energy efficiency components in gross regional product (GRP) and the key products, defines the impact of economic activities and local projects on the dynamics of energy consumption, and identifies trends in target 
indicators. Taking into account the information characterizing social and economic development of a region and development programmes for the local fuel and energy sector, energy balance projections make it possible to detect potential risks to the region's energy supply.

The issue of credibility of fuel and energy balance plan as a complex information system is quite significant. The authors do not consider their proposed approach to be the best or highly satisfactory one. Nor do they aspire to find the most accurate and reliable solution to a highly unconventional task - that of designing the best method of providing a detailed elaboration of and alignment between the current and projected parameters of the region's social and economic development forecasts and its fuel and energy balance plan from a number of available options. The authors base their conclusions on their calculations and empirical framework of the fuel and energy balance plans that they drafted and adjusted between 2007 and 2013, as well as available statistics for 2004-14 and the evolving methods of forming fuel and energy balance plans that they [4] and other scholars [5-9] have used. They believe that it will be useful to discuss these research undertakings to gradually reduce the significance of information and methodological problems.

\section{INFORMATION AND METHODOLOGICAL ASPECTS OF FORMING A FUEL AND ENERGY BALANCE PLAN}

Available statistics do not allow for a detailed analysis of distribution of fuel and energy resources across the full list of economic uses. For example, the structure of electricity consumption is only partially described in open sources. Rounding of data in the information database of the Federal State Statistics Service (Rosstat) creates additional errors that erode value added in some economic activities to zero. As a result, a problem arises of comparing economic activities by the energy intensity of value added.

The core idea of the author's approach to forming fuel and energy balance plans is to use the Rosstat-provided detailed GRP broken down by economic activity, to analyse the dynamics of the obtained vectors between 2004 and 2013 and to forecast value added and energy intensity for each economic activity.

Given the above-mentioned issues, the method applied by the authors for decomposing GRP in the previous and current years and for making projections takes into account the following structural features of the available information base:

- estimated GRP for the current year, as computed in the regional social and economic development forecast. The available data for the past year include the total heat and electricity consumption by small enterprises, households as well as by medium-sized and large companies (for a reduced list of economic activities);

- detailed data on fuel consumption by households, medium-sized and large enterprises are available;

- information is available about the structure of turnover for basic economic activities and the characteristics of the fuel and energy balance plans for the period starting in 2007 and the indicators of GRP for the period starting in 2004;

- a volume of historical information starting from 2007 has been obtained on the trends in the output of key products, individual and aggregate energy intensity and the dynamics of the target indicators of energy efficiency and energy conservation.

We should also note fatal asymmetry in procedures of matching the parameters of fuel and energy balance and forecasts of social and economic development. The approved scenario indicators of social and economic development, in addition to the energy consumption characteristics, could not 
be the subject to adjustments in coordination procedures. Therefore, the reliability of approved fuel and energy balance depends on the quality of the region development forecast. In practice authors have also formed an additional fuel and energy balance embodiment, based on their own analysis and estimates of the dynamics of the certain economic activities development.

\section{CALCULATION AND ANALYSIS}

Given the above, the following calculations are performed.

To add structure to the formally assessed GRP in the previous and current years, historical information is analysed. Relationship is assessed between the GRP at basic prices $\left(\boldsymbol{G R} \boldsymbol{P}_{c}\right)$ and the gross turnover (TURN) of medium-sized and large companies for the period between 2004 and 2013. An analysis of correlations between a time series of GDP and the turnover in the region and between value added by activity $\left(\boldsymbol{V A} \boldsymbol{L}_{d c j}\right), \boldsymbol{j}=\overline{\mathbf{1 , 1 5}}$ and turnover $\left(\boldsymbol{T} \boldsymbol{U} \boldsymbol{R} \boldsymbol{N}_{j}\right)$ shows that the correlation coefficient in the first case exceeds 0.87 . Obviously, the correlation coefficient is partially explained by the fact that both indicators depend on a common factor - that of the positive economic environment. Regression analysis of the dependence $\boldsymbol{G R} \boldsymbol{P}_{\boldsymbol{c}}=\boldsymbol{F}(\boldsymbol{T} \boldsymbol{U} \boldsymbol{R})+\boldsymbol{\varepsilon}$ confirms, to a high degree of approximation (normalized $\boldsymbol{R}^{2}=0.86$ ), a relationship between the indicators: $\boldsymbol{G R P}_{\boldsymbol{c}}=\boldsymbol{C o n s t}+\boldsymbol{K}^{*} \boldsymbol{T U} \boldsymbol{R} \boldsymbol{N}+\boldsymbol{\varepsilon}$, with the confidence interval containing actual and projected GRP volumes for the past 2 years. A similar correlation coefficient for individual economic activities is also quite high, so the merit of assessing the GRP and its structure on the basis of statistical relationships between the target indicators and the actual turnover data is determined by more than their definitions. It is useful to compare the confidence interval $(\underline{\boldsymbol{G R P}} \boldsymbol{c}, \overline{\boldsymbol{G R P}} \boldsymbol{c})$ with the projected GRP for the current year from the officially approved forecast of social and economic development.

The basic method of decomposing GRP that is based on the 'value added-to-turnover' ratio for the current year compared to that for the prior year and the subsequent 'normalization' of the indicators to ensure GRP balance is accurate enough, provided that the ratio exhibits high inertia during the last few years of the retrospective period. It is, therefore, advisable to use regression and time trend analysis for assessing the dependence of value added on turnover.

To make a conversion from basic prices to constant prices and to calculate chain-linked indices of physical volume, value-added deflators need to be computed for each type of economic activity. Information available for analysis includes actual deflators for the considered period and operational statistics. Regression analysis takes into account projected GRP at current basic prices from the officially approved forecast of social and economic development and the index of the physical volume of GRP for each scenario. A settlement procedure is implemented to determine a non-contradictory structure of the GRP.

It is impossible to create a method that would produce the most accurate prediction interval because of incomplete information. Relying on the officially approved projections of the region's social and economic development is not entirely reasonable because of the abridged structure of GRP. Moreover, current scenarios might differ significantly from observed trends. Our calculations show, though, that statistical analysis methods can ensure the consistency and balance of estimates as well as their relative accuracy.

Breaking down GRP by economic activity makes it possible to calculate the indices of trends in energy consumption, energy conservation and energy efficiency for the base year versus the prior year. At the same time, chain-linked and aggregate deflators (against 2007) are refined for the period starting 2004.

It is advisable to apply rigorous (formal) methods for calculating GRP parameters. Assume that $t$ is the year index (in $\boldsymbol{t} \in \overline{\mathbf{2 0 0 4} ; \mathbf{2 0 1 3}}$ ), $\boldsymbol{b}$ is the index of the first (base) year, $\boldsymbol{G R} \boldsymbol{P}_{t c}$ is the GRP at cur- 
rent basic prices, $\boldsymbol{G R P}_{\boldsymbol{b c}}$ is the base year's GRP at current basic prices; $\boldsymbol{G R P}_{t \boldsymbol{s}}$ и $\boldsymbol{G} \boldsymbol{R} \boldsymbol{P}_{\boldsymbol{b} \boldsymbol{s}}$ are similar indicators at constant prices. The following equation emerges for the baseline year $\boldsymbol{G} \boldsymbol{R} \boldsymbol{P}_{\boldsymbol{b s}}=\boldsymbol{G} \boldsymbol{R} \boldsymbol{P}_{\boldsymbol{b}}$. In the Rosstat data the first year (2004) is considered to be the base year and chain-linked indices of the physical volume of GRP (at prior year's prices) $\boldsymbol{\alpha} t \mathbf{s}$ are shown. For $\boldsymbol{t}=\boldsymbol{b}$ we have $\mathrm{a}_{\boldsymbol{b} \boldsymbol{s}}=1$ (or $100 \%$ ); consequently, $\boldsymbol{G R} \boldsymbol{P}_{t s}$ - the physical volume of GRP in the year $\boldsymbol{t}$ at constant prices of the first year $(\boldsymbol{b}=2004)-$ is defined by the equation

$$
G R P_{t s}=\prod_{t=b}^{t} \alpha_{t s} \times G R P_{b c}
$$

or by the sequence of operations

$$
G R P_{t s}=G R P_{(t-1) s} \times a_{t s} ; t=\overline{2005 ; 2013}
$$

Chain-lined deflator indices $\boldsymbol{\beta}_{t h}$ for the year 2004 are computed as follows:

$$
\beta_{t h}=\frac{G R P_{t c}}{G R P_{(t-1) c} / \alpha_{t s}} ; t=\overline{2005 ; 2013}
$$

Aggregate deflator indices $\boldsymbol{\beta}_{\boldsymbol{t} \text { int } \boldsymbol{b}}$ for the year 2004 are derived as:

$$
\beta_{\text {tint } b}=\frac{G R P_{t c}}{G R P_{b s}} ; t=\overline{2005 ; 2013}
$$

or by chain-linking:

$$
\beta_{\text {tint } b}=\prod_{t=b}^{t} \beta_{t h} ; t=\overline{2005 ; 2013}
$$

Russian regulations for calculating energy efficiency and energy conservation accept 2007 as the base year. The formula for computing aggregate deflators to the new base year $\left(b_{1}=2007\right)$ in our case (when aggregate deflators relative to the first year of the period are already known) is given by

$$
\beta_{\text {tint } b_{1}}=\frac{\beta_{\text {tint } b}}{\beta_{b_{1} \text { int } b}} ; t=\overline{2004 ; 2013}
$$

Similar operations are performed for each type of economic activity for the entire time period, including the current year when information is incomplete. The structural parameters of GRP by economic activity, including the evolution of the chain-linked and aggregate added value deflators adjusted to the start of the period and the baseline year of 2004, are refined in line with the scenarios. This information is essential for verifying the accuracy of calculations. More specifically, a check is needed on the GRP ratio at current and constant prices of the base year.

$$
G R P_{t c}=\sum_{j} \text { AddedValue }_{t c j} ; G R P_{t s}=\sum_{j} \text { AddedValue }_{t s j} ; j=\overline{1 ; 15}
$$

where $_{\text {AddedValue }}$ tcj ; AddedValue $_{t s j}, j=\overline{1 ; 15}$ is value added at current and adjusted prices by economic activity. The calculations of deflators are also verified for accuracy and error. 


$$
G R P_{t s}=G R P_{t c} \times \bar{\beta}_{t i n t b} ; t=\overline{2004 ; 2013}
$$

$\overline{\boldsymbol{\beta}}_{t \text { int } b}$ is chain-linked GRP deflator, computed as a mean for all types of economic activity.

$$
\bar{\beta}_{\text {tint } b}=\sum_{j} \beta_{\text {tint }_{j}} \times \delta_{t j} ; j=\overline{1 ; 15}
$$

where $\boldsymbol{\delta}_{t j}=$ AddedValue $_{t c j} / \boldsymbol{G R P} \boldsymbol{P}_{t c} ; \boldsymbol{t}=\overline{2004 ; 2013} ; j=\overline{1 ; 15}$ is the share of the economic activity in the region's GRP (at current prices).

It is essential to verify the Rosstat data to account for rounding errors in the structure of GRP by economic activity. Because of the rounded shares of economic activities in GRP, the sum total of value added at current prices does not coincide with the corresponding GRP volumes for the region. The rounding of physical volume indices for economic activities also upsets the balance of GRP reporting by economic activity and value added. The adjustment procedures for current and constant prices are identical for each year of the historical period. The following formal description of data adjustment at 2007 constant prices implies that appropriate adjustments in current prices have already been made.

We shall use the following notation: $\gamma_{j t}$ are the indices of the physical volume of value added by economic activity $\boldsymbol{j}=1,2, \ldots, 15 ; t=\underline{2008,2012}$ is the year index; $1_{j t}$ are the required adjustment factors (their values are found in a neighbourhood of 1 ); $1_{j t}^{*} \gamma_{j t}$ are the target adjustment results; $\boldsymbol{d}_{j t}$ are the initial value-added volumes, $\boldsymbol{d}_{j t}=\boldsymbol{d}_{j t-1} * \gamma_{j t}$, for the base year $\mathrm{t}=2007 \boldsymbol{d}_{\boldsymbol{j} 2007}$ is the known constant value at current prices.

Criteria in the procedures are written as:

$$
F_{t}=\sum_{j=1}^{j=15}\left(\lambda_{j t} \times \gamma_{j t}-\gamma_{j t}\right)^{2} \rightarrow \min , t=\overline{2008 ; 2012}
$$

Variables to be optimized are $\lambda_{j t}, \boldsymbol{j}=1,2, \ldots, 15$.

The restriction is that balancing conditions (11) should be met for each year in the historical period (the procedure is unnecessary for the base year):

$$
\sum_{j=1}^{j=15} d_{j t}=\sum_{j=1}^{j=15} \lambda_{j t} \times \gamma_{j t} \times d_{j t-1}=G R P_{t s}, t=\overline{2008 ; 2012}
$$

It provided that all built-in associations among value-added measures in MS Excel calculations are observed.

The historical data are only adjusted once or when updated by Rosstat. The operations are easily automated using MS Excel tools, and the method can also be used for normalizing (balancing) the structural parameters of GRP when assessing its projected structure. The approach imitates the maximum likelihood method (the rounding of yielded results does not contradict the Rosstat data).

A general pattern for producing fuel and energy balance forecasts combines two key approaches:

- the balance plan should reflect the regional production profile and the energy intensity of the area's key products and services (production section of the balance plan);

- the balance plan should indicate the structure of energy consumption by economic activity, making it possible to take into account the sectoral scope of the balance plan. 
A forecasting method based on product aggregation only proves ineffective because many areas of economic activity such as health care, education, financial services, public administration, science and culture are not included in statistics in a comprehensive enough way. Such information is fragmented and is prone to change.

Statistical estimates of added value indicators were normalized in line with known projected GRP values for the region, and correlation between formally approved scenarios and confidence interval estimates was analysed. The share of each type of economic activity in the region's GRP was subsequently determined.

Factor regression analysis or methods of time series analysis were used to produce a forecast of value added at constant prices for the year 2007 and the energy intensity of each type of economic activity. The most suitable regression equations were selected according to the approximation criterion (mostly, power laws, logarithmic dependence and autocorrelation) that did not contradict the expert logic of the region's economic development. Expert stabilization factors or exponential smoothing were applied to time series data that exhibited spikes, breaks or drops closer to the end of the historical period. The method is to be further elaborated to include, among other things, price trends. To forecast fuel and energy consumption by households, regression was used with the 'time' variable and the 'floor area per person' variable; to produce a forecast for losses of gas, power and heat in transmission and for self-consumption, regressions were used with the 'time' variable and 'distribution' variable.

Comparing forecast trends in GRP energy intensity with target values of appropriate indicators makes it possible to assess the attainability of the targets. For example, total energy consumption $\Delta$ EnergCost $\boldsymbol{t}_{\boldsymbol{t}}$ for any year $\boldsymbol{t}$ during the forecast period and the impact of structural shifts in GRP $\boldsymbol{\delta}$ EnergCost $\boldsymbol{t}_{\boldsymbol{t}}$ may be assessed with the formulas (12) and (13).

$$
\begin{gathered}
\bar{\Delta} \text { EnergCost }_{t}=\sum_{j=1 ; 15} \text { AddedValue }_{t s j} \times\left(\text { EnergCost }_{2007 j}-\text { EnergCost }_{t j}\right) \\
\delta \text { EnergCost }_{t}=\sum_{j=\overline{1 ; 15}}\left(\delta_{t j}-\delta_{2007 j}\right) \times \text { EnergCost }_{2007 j} ; t=\overline{2013 ; 2017}
\end{gathered}
$$

\section{CONCLUSION}

Estimates have shown that the found confidence intervals for energy consumption indicators considerably exceed their variation when observed retrospectively. That is why, the officially approved 'optimistic' forecasts of GRP and its structure can be interpreted as finding the midrange. The conditional probability of formal estimates falling within the fixed limits of projected variation depends on the degree of bias in forecasts of social and economic development.

Unlike the guidelines of the IEA, Eurostat and the UN, the blueprint for fuel and energy balance plans in the regions of Russia breaks down energy consumption in industry, agriculture, transport and housing according to products, services, processes and types of work. The fuel and energy balance concept used by the authors distinguishes a set of key products that determine the industrial profile of the region, making it possible to reflect the physical indicators of energy efficiency that are not influenced by price distortions in GRP and added value [10].

Incomplete and imprecise information is only partly to blame for increasing the uncertainty and unreliability of fuel and energy balance plans. But it is information-related problems that create fundamental obstacles to developing and improving methods that could produce reliable forecasts. 
It is important that the information problems should be addressed at the legislative level. For example, data forms should be designed that would match up with the types and structure of fuel and energy balance plans in regions and municipalities; deadlines for forming fuel and energy balance plans and their publication should be agreed upon.

\section{ACKNOWLEDGEMENT}

The work was supported by Act 211 Government of the Russian Federation, contract № 02. A03.21.0006.

\section{REFERENCES}

[1] World Energy Outlook, available at http://www.iea.org/bookshop/700-World_Energy_ Outlook_2015

[2] Federal law № 282, On official statistical accounting and state statistics system in the Russian Federation of 29 Nov 2007 (edited 23 Jul 2013), [in Russian], available at http://www.consultant.ru/document/cons_doc_LAW_72844/

[3] Federal law № 172, On strategic planning in the Russian Federation of 28 Jun 2014, [in Russian], available at http://www.rg.ru/2014/07/03/strategia-dok.html

[4] Danilov, N., Silin, V., Dobrodey, V. \& Popov, V., Energy problems of the rational use of the economic potential of the region. WIT Transactions on Ecology and the Environment, 190, pp. 419-423, 2014.

http://dx.doi.org/10.2495/EQ140401

[5] Fuel and energy balance plans, [in Russian], available at http://www.eriras.ru/data/4/rus.

[6] Bashmakov, I.A., Siniak, YV. \& Makarov, A.A., Costs and Benefits of Low-Carbon Economy and Social Transformation in Russia, [in Russian], Centre for Effective Energy Use: Moscow, $178,2014$.

[7] Bashmakov, I. \& Myshak, A., Factor analysis of evolution of russian energy efficiency: metodology and outcomes, [in Russian]. Problems of Economics, 10, pp. 117-131, 2012.

[8] Brandoni, C. \& Polonara, F., The role of municipal energy planning in the regional energyplanning process. Energy, 48, pp. 323-338. 2012. http://dx.doi.org/10.1016/j.energy.2012.06.061

[9] Sampaio, H.C., Dias, R.A. \& Balestieri, J., Sustainable urban energy planning: the case study of a tropical city. Applied Energy, 104, pp. 924-935, 2013.

http://dx.doi.org/10.1016/j.apenergy.2012.12.022

[10] Tracking Industrial Energy Efficiency and $\mathrm{CO}_{2}$ Emissions, OECD/IEA: Paris, 321, 2007. 